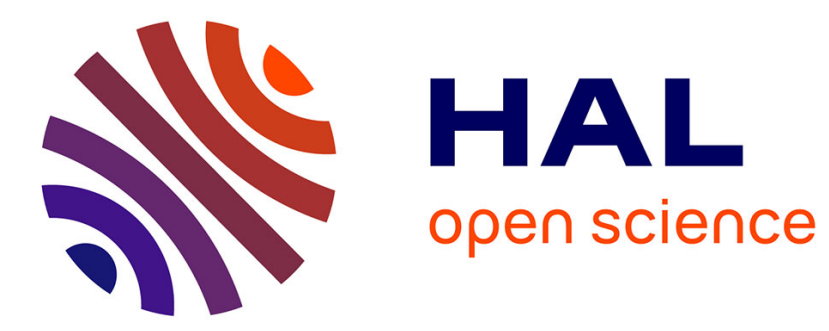

\title{
Spatial regularization based on dMRI to solve EEG/MEG inverse problem
}

Brahim Belaoucha, Théodore Papadopoulo

\section{To cite this version:}

Brahim Belaoucha, Théodore Papadopoulo. Spatial regularization based on dMRI to solve EEG/MEG inverse problem. EMBC 2017 - 39th Annual International Conference of the IEEE Engineering in Medicine and Biology Society, Jul 2017, Jeju, South Korea. hal-01533396

\section{HAL Id: hal-01533396 \\ https://hal.inria.fr/hal-01533396}

Submitted on 6 Jun 2017

HAL is a multi-disciplinary open access archive for the deposit and dissemination of scientific research documents, whether they are published or not. The documents may come from teaching and research institutions in France or abroad, or from public or private research centers.
L'archive ouverte pluridisciplinaire HAL, est destinée au dépôt et à la diffusion de documents scientifiques de niveau recherche, publiés ou non, émanant des établissements d'enseignement et de recherche français ou étrangers, des laboratoires publics ou privés. 


\title{
Spatial regularization based on dMRI to solve EEG/MEG inverse problem
}

\author{
Brahim Belaoucha*, and Théodore Papadopoulo
}

\begin{abstract}
In this paper, we present a new approach to reconstruct dipole magnitudes of a distributed source model for magnetoencephalographic (MEG) and electroencephalographic (EEG). This approach is based on the structural homogeneity of the cortical regions which are obtained using diffusion MRI (dMRI). First, we parcellate the cortical surface into functional regions using structural information. Then, we use a weighting matrix that relates the dipoles' magnitudes of sources inside these functional regions. The weights are based on the region's structural homogeneity. Results of the simulated and real MEG measurement are presented and compared to classical source reconstruction methods.
\end{abstract}

\section{INTRODUCTION}

EEG and MEG are two imaging modalities that provide information about brain activity with high temporal resolution. The sources of MEG/EEG are current sources that are modeled using dipoles which are distributed in the cortical surface. Obtaining the distributed sources activation from these measurements is an underdetermined problem due to the small number of measurements with respect to the number of distributed sources. To obtain a unique solution, different constraints can be used [1]. They can be divided into three different categories: spatial, temporal, and spatiotemporal constraints. In our work, we focus on spatial constraints. Minimum norm estimate (MNE) was introduced to obtain a unique source reconstruction [2, 3]. MNE gives a linear solution which makes it a simple and attractive approach. But it suffers from overestimation of the extents of the active regions. To address these limitations, many MNE variants have been proposed by introducing different weighting matrices [1]. But all neglect the structural information which has been shown to be in relation to the functional homogeneity of brain regions by several studies [4]-[8]. In Philippe et al. [9], the authors used a weighting matrix as a penalty term which favour constant activation per region $(\mathrm{CP})$. In their work, they do not consider the possible structural inhomogeneity.

dMRI is the only non-invasive imaging modality that allows the access to the anatomical connectivity. That is why it has been used to parcellate the cortical surface [7, 10]. In this work, we present another variant of the weighted minimum norm in which the elements of the weighting matrix depend on the structural homogeneity of the regions obtained from dMRI. We call it structural weighted minimum norm

This work received funding from ERC under the European Union's Horizon 2020 research and innovation program (ERC Advanced Grant agreement No 694665 : CoBCoM) and French ANR contract Vibrations. Both authors are with the Université Côte d'Azur, Inria, Nice, France (email: brahim.belaoucha@inria.fr, theodore.papadopoulo@inria.fr). estimate (sWMNE). The proposed method is tested using synthetic data and is compared to some of the reconstruction algorithms that can be found in the literature. Also, real data was used to test the accuracy of the algorithm. The results were compared with the results of functional MRI (fMRI) group analysis study with the same subjects performing the same tasks [11].

\section{DATA ACQUISITION AND PROCESSING}

Structural and diffusion MRI data were taken from Wakeman et al. [11]. The $1 \mathrm{~mm}$ isotropic resolution T1 weighted images of size $256 \times 256 \times 192$ were acquired by a Siemens $3 \mathrm{~T}$. The $2 \mathrm{~mm}$ isotropic resolution diffusion weighted images of size $96 \times 96 \times 68$ were collected by the same scanner (64 gradient directions and $b$-value $\left.=1000 \mathrm{~s} / \mathrm{mm}^{2}\right)$, with one b0 image (an image without diffusion gradient). The cortical surface was extracted from T1, using Freesurfer [12], and re-meshed to $10^{4}$ vertices (sources). The transformation between the anatomical and diffusion space was obtained by using FSL [13].

MEG (102 magnetometers, 204 planar gradiometers) and EEG (70 electrodes) were measured simultaneously in a magnetically shielded room. The face stimuli contain three sets of 450 gray scale photographs, one third of unfamiliar people (unknown to the participants), one third of famous people and the remaining are of scrambled faces. The reader is referred to [11] for more details.

In this work, we are interested in localizing face recognition areas. For this reason, we use only the measurement acquired when using photos of famous people subtracted to the ones obtained when using scrambled faces. Low pass filter of cut-off frequency $45 \mathrm{~Hz}$ was used to smooth the data. The MEG/EEG forward problem, lead field matrix $\mathbf{G}$, is obtained using OpenMEEG [14, 15].

\section{CORTICAL PARCELLATION}

The mutual nearest neighbor (MNN) parcellation algorithm divides the cortical surface of $N$ points into regions with the highest homogeneity according to a similarity measure [10]. MNN uses connectivity profiles of sources to parcellate the cortex substrate. Connectivity profile of a source is a vector containing the probability of the existence of anatomical connections between the source and the other image voxels. This probability is obtained by running a probabilistic tractography using FSL [13]. MNN depends on one parameter, $s$, that controls the resulting number of regions. We use Tanimoto [16] measure to quantify the 
similarity between the connectivity profiles. The Tanimoto similarity measure between source $i$ and $i$ is:

$$
d_{i, j}=\frac{X_{i} \cdot X_{j}}{X_{i} \cdot X_{j}+\left\|X_{i}-X_{j}\right\|_{2}^{2}}
$$

where $X_{i}$ and $X_{j}$ is the vector form of the connectivity profile of source $i$ and $j$ respectively. The Tanimoto is symmetric and has values between 0 and 1. The MNN can result to small regions due to the structural inhomogeneity. The small regions, regions with less than $\frac{N}{s}$ sources, were merged with valid regions in a way that give the highest mean similarity value.

\section{MEG/EEG INVERSE PROBLEM}

In a distributed source model with $m$ sources and $n$ sensors, the EEG/MEG measurement is related to the sources' magnitudes by the following linear relation:

$$
\mathbf{M}=\mathbf{G S}+\epsilon
$$

where $\mathbf{M} \in \mathbb{R}^{n \times T}$ is the measurement ( $T$ number of time samples), $\mathbf{G} \in \mathbb{R}^{n \times m}$ is the lead field matrix that contains the contribution of sources to each sensor and $\mathbf{S} \in \mathbb{R}^{m \times T}$ is a matrix that contain the sources' magnitudes. Finally, the measurement is corrupted by an additive Gaussian noise of a zero mean, $\epsilon$. The minimum norm estimates sources' magnitudes by optimizing the following functional:

$$
U(\mathbf{S})=\|\mathbf{M}-\mathbf{G S}\|_{2}^{2}+\lambda\|\mathbf{S}\|_{2}^{2}
$$

where $\|\cdot\|$ is $l_{2}$ norm and $\lambda(\geq 0)$ is the trade-off parameter between the prior and the data fit term. The solution of Eq (2) is:

$$
\mathbf{S}_{m n e}=\left(\mathbf{G}^{t} \mathbf{G}+\lambda \mathbf{I}\right)^{-1} \mathbf{G}^{t} \mathbf{M}
$$

where $\mathbf{X}^{\mathbf{t}}$ is the transpose of matrix $\mathbf{X}$ and $\mathbf{I}$ is $m \times m$ identity matrix. Let's define the following functional:

$$
U(\mathbf{S})=\|\mathbf{M}-\mathbf{G S}\|_{2}^{2}+\mu\|\mathbf{W S}\|_{2}^{2}+\lambda\|\mathbf{S}\|_{2}^{2}
$$

where $\mathbf{W}$ is a weighting matrix and $\mu(\geq 0)$ is a regularization parameter. We propose to use a weighted matrix whose elements are defined by similarity values of the connectivity profile vectors (see section III). We consider $\mathbf{W}$ to be a block matrix in which each block corresponds to a cortical region defined by:

$$
S_{i} \in R_{p} \rightarrow \mathbf{W}(i, j)= \begin{cases}1-\frac{1}{d_{i}} & \text { if } i=j \\ -\frac{d_{i, j}}{\sqrt{d_{i} d_{j}}} & \text { if } S_{j} \in R_{p} \\ 0 & \text { if } S_{j} \notin R_{p}\end{cases}
$$

where $S_{i}$ and $R_{p}$ are, respectively, the $i^{t h}$ source and $p^{t h}$ cortical region. $d_{i, j}$ is the similarity value between the connectivity profile vector of source $i$ and $j$.

$$
d_{i}=\sum_{j=1}^{\left|R_{p}\right|} d_{i, j}
$$

where $\left|R_{p}\right|$ is the number of sources in the $p^{t h}$ region.

To have an idea about the effect of this weighting matrix, let's assume that we have five sources. $P=[1,1,1,2,2]$ is

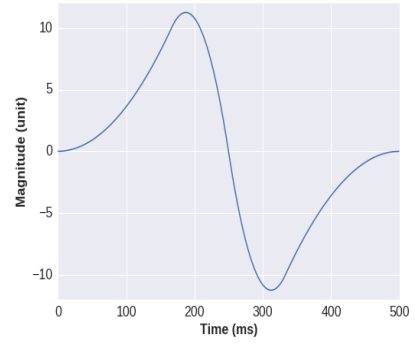

(a)

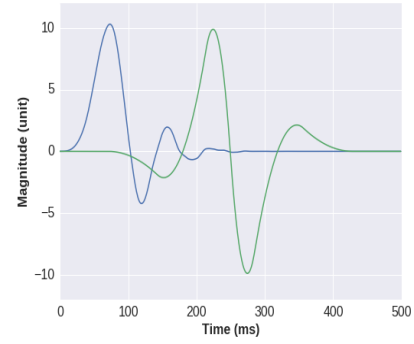

(b)
Fig. 1: Simulated data used to activate one region (a) and two regions (b).

a vector containing the label of each source. The weighting matrix, $\mathbf{W}$, for this case is:

$$
\mathbf{W}=\left(\begin{array}{ccccc}
1-\frac{1}{d_{1}} & \frac{-d_{1,2}}{\sqrt{d_{1} d_{2}}} & \frac{-d_{1,3}}{\sqrt{d_{1} d_{3}}} & 0 & 0 \\
\frac{-d_{1,2}}{\sqrt{d_{1} d_{2}}} & 1-\frac{1}{d_{2}} & \frac{-d_{2}, 3}{\sqrt{d_{2} d_{3}}} & 0 & 0 \\
\frac{-d_{1,3}}{\sqrt{d_{1} d_{3}}} & \frac{-d_{2,3}}{\sqrt{d_{2} d_{3}}} & 1-\frac{1}{d_{3}} & 0 & 0 \\
0 & 0 & 0 & 1-\frac{1}{d_{4}} & \frac{-d_{4,5}}{\sqrt{d_{4} d_{5}}} \\
0 & 0 & 0 & \frac{-d_{4,5}}{\sqrt{d_{4} d_{5}}} & 1-\frac{1}{d_{5}}
\end{array}\right)
$$

$\|\mathbf{W S}\|_{2}$ is minimum when:

$$
J_{2}=\sqrt{\frac{d_{2}}{d_{1}}} J_{1}, \quad J_{3}=\sqrt{\frac{d_{3}}{d_{1}}} J_{1}, \quad J_{4}=\sqrt{\frac{d_{4}}{d_{5}}} J_{5}
$$

If source $i$ and $j$ are in the same region, $\|\mathbf{W S}\|_{2}$ constrains $i$ and $j$ to have activations related by the following relationship $S_{j} \approx \sqrt{\frac{d_{j}}{d_{i}}} S_{i}$. The solution of Eq (4) is:

$$
S_{\text {swmne }}=\left(\mathbf{G}^{t} \mathbf{G}+\mu \mathbf{W}^{t} \mathbf{W}+\lambda \mathbf{I}\right)^{-1} \mathbf{G}^{t} \mathbf{M}
$$

Each block of $\mathbf{W}$ corresponds to a Laplacian weighted by the similarity measure values of each region. The method explained in Philippe et al. [9], that we call CP, can be obtained from our method by replacing all the $d_{i, j}$ 's by 1. The regularization parameters are obtained using crossvalidation [17].

\section{RESUlTS AND DisCUSSION}

\section{A. Simulation setup}

For reasonable fast computation time and comparison between sWMNE, MNE and CP, we generated a simulation with 20 sensors and 500 points distributed equally into 50 regions i.e. 10 points in each region. For each region, $R_{i}$, we generate randomly, from normal distribution $\mathcal{N}(100,200)$, the mean structural connectivity vector $\left(r_{i}\right)$ of length equal to $10^{3}$. We give to a source point $j$, that belongs to $R_{i}$, the following connectivity profile vector:

$$
r_{j}=r_{i}+\epsilon_{j}
$$

where the elements of the vector $\epsilon_{j}$ are obtained randomly from $\mathcal{N}(50,100)$, values lower than 100 were set to zero. The elements of the weighting matrix are obtained by computing the Tanimoto similarity measure between the sources' connectivity vectors that belong to the same regions. The forward model $G$, gain matrix, is drawn from a $\mathcal{N}(0,1)$. We consider two test configurations: 


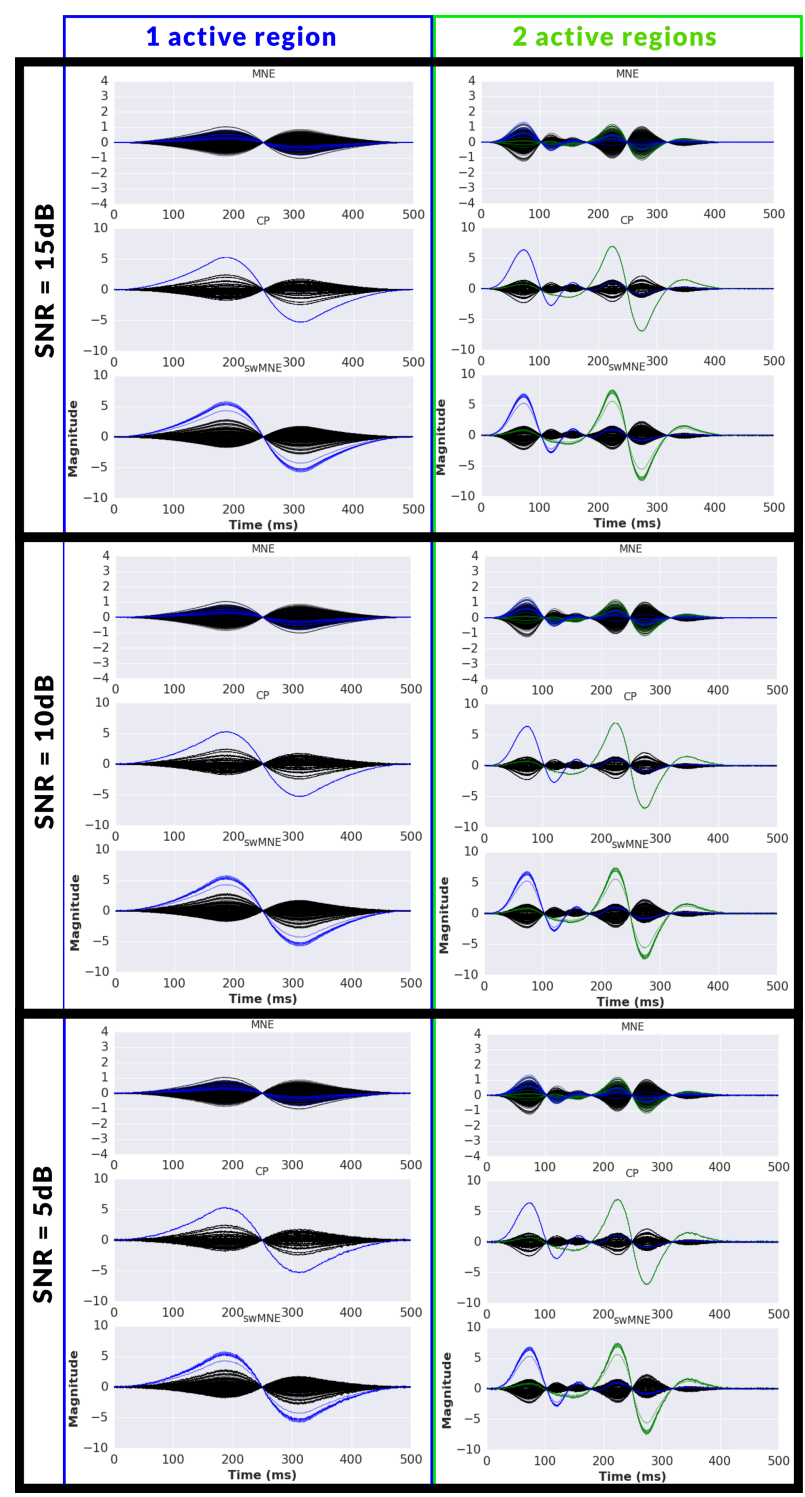

Fig. 2: Mean (over 100 runs) reconstruction using MNE, CP and sWMNE at different noise levels $(\mathrm{SNR}=15,10,5 \mathrm{~dB})$. Left panel: Results of the $1^{\text {st }}$ test configuration. Right panel: results of the $2^{\text {nd }}$ test configuration. We show in color, blue or/and green, the reconstructed magnitudes of the simulated region(s) and in black the remaining sources.

- One active region (see Figure 1 (a)).

- Two active regions (see Figure 1 (b)).

In the first test configuration, we activate 20 random regions. For each active region, we run the reconstruction algorithms 100 times at three different noise levels (Signal-to-Noise ration $(\mathrm{SNR})=\{15,10,5\} \mathrm{dB})$. In the second, we activate 20 random pairs of regions. For each pair of regions, we run the reconstruction algorithms 100 times at the same noise levels mentioned earlier. Figure 2 shows an average, one of the 20 randomly activated regions in both test configurations, the reconstructed sources using MNE, CP and sWMNE. The left and right panels correspond, respectively, to the results

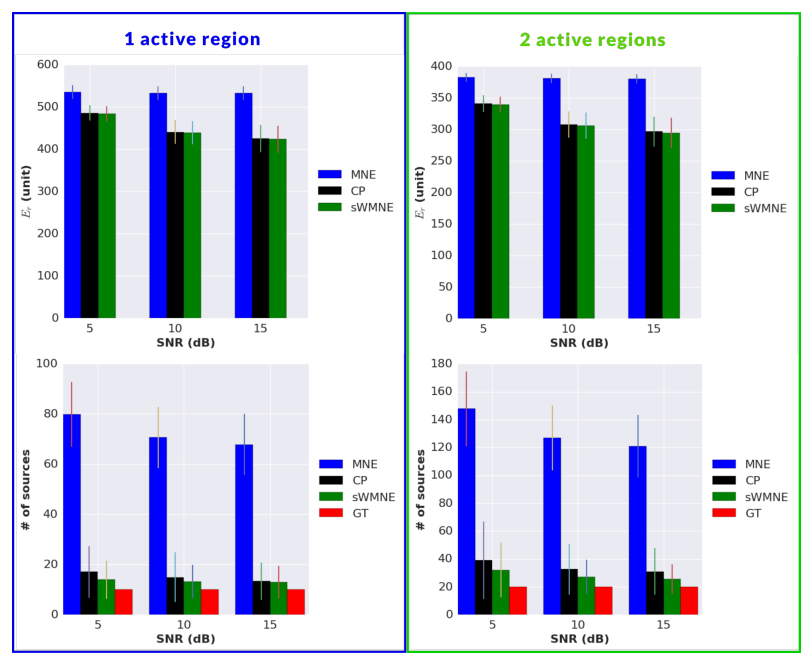

Fig. 3: Top raw: reconstruction error $E_{r}$ of the different algorithms. Bottom, the number of sources that have $l_{2}$-norm $\geq 25 \%$ of the highest energy in the source space. The results are obtained for different noise levels. GT, in red, refers to ground truth.

when activating one and two regions. To test the accuracy of the reconstructions, we compute the source reconstruction error $\left(E_{r}\right)$ :

$$
E_{r}\left(S_{g t}, S_{r}\right)=\left\|S_{g t}-S_{r}\right\|_{2}
$$

where $S_{g t}$ and $S_{r}$ are, respectively, the simulated (ground truth) and reconstructed sources, see the upper part of Figure 3 . Also, we test the focality of the three methods by counting the number of sources that have activations higher than $25 \%$ of the highest activation, see the lower part of Figure 3. A degree of activation of a source $i$ is computed as the $l_{2}$ norm of the reconstructed time course of $i$.

The MNE smears the activation which makes it hard to localize spatially the active regions, see Figure 2 . In the $\mathrm{CP}$ and sWMNE, we can distinguish from the reconstructions, see Figure 2, which regions are more active. sWMNE is more flexible than $\mathrm{CP}$ by allowing magnitude variation due to a difference of the structural connectivity between sources of the same region. sWMNE does not show significant improvement in terms of $E_{r}$, see Figure 3, with compared to $\mathrm{CP}$ but the number of active sources obtained from sWMNE is closer to the ground truth.

\section{B. Real data}

We tested sWMNE using real MEG data obtained from [11] which was presented briefly in section II. In this paper, we show only the results of one subject due to page limitation. In Figure 4, we show the normalized $l_{1}$ norm of the source reconstructed intensities using sWMNE (left) and MNE (right) between time $t=0$, the onset, and $t=$ $0.6 \mathrm{~s}$ thresholded at $15 \%$. In the contrary to MNE, sWMNE provides more focal reconstruction.

In the sWMNE, we can distinguish fusiform and occipital activation in both hemispheres. Also in all the eleven subjects 

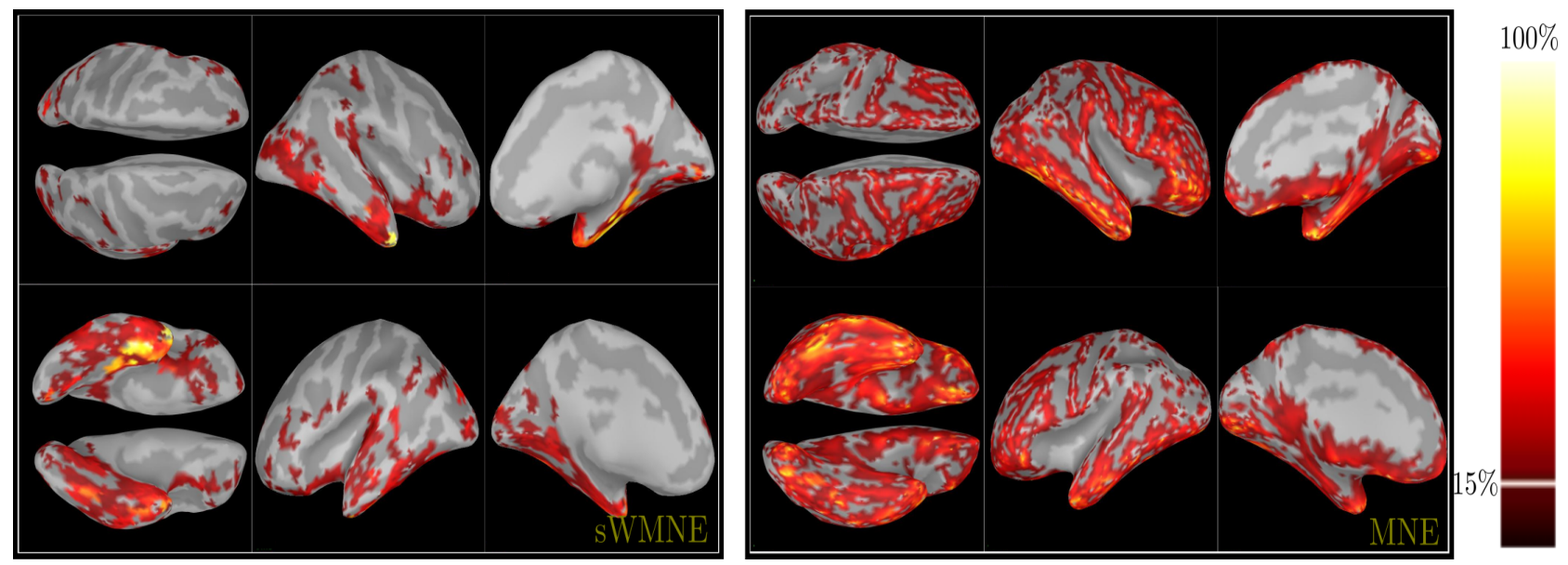

Fig. 4: The $l_{1}$-norm of the reconstructed sources, from MEG [11], of Subject 1 displayed on the inflated surface. We show only values above $15 \%$ of the highest $l_{1}$-norm. The $s$ parameter of MNN was set to 800 .

in [11], anterior temporal lobe and inferior pre-frontal were found to be active. Orbitofrontal cortex was found to be more active in subject 4,9 and 11 . The right temporal pole of subject 1 and 2 were found to be more active than the left one. These regions coincide with what was reported in Wakeman et al. [11] in which they used the same paradigm and participants to acquire fMRI. The study whether some of these regions are dedicated to human faces [18] or intervene in other visual stimuli [19] is outside the scope of this work.

sWMNE shows better results, in terms of reconstruction error and focality, with compared to MNE. We observed no significant difference in the reconstruction error between $\mathrm{CP}$ and sWMNE but the latter gives a better approximation of the activation extent.

\section{CONCLUSION}

This paper presented a method to use information from dMRI to solve the EEG/MEG inverse problem. A weighting matrix whose elements are obtained from computing the similarity between the connectivity vector pairs inside each region is used to regularize the inverse problem. This matrix allows sources to differ according to their structural homogeneity. Finally, the source reconstruction and cortical surface parcellation depend on the similarity measure used to quantify the structural homogeneity between the different connectivity profiles. Other similarity measures can be used and their effect on the reconstruction should be investigated.

\section{ACKNOWLEDGMENTS}

We thank Dr. Daniel Wakeman and Prof. Richard Henson for providing the raw data.

\section{REFERENCES}

[1] R. Grech, , T. Cassar, J. Muscat, et al., "Review on solving the inverse problem in EEG source analysis," Journal of NeuroEngineering and Rehabilitation, vol. 5, no. 1, pp. 1-33, 2008.

[2] J. Z. Wang, S. J. Williamson, and L. Kaufman, "Magnetic source images determined by a lead-field analysis: the unique minimumnorm least-squares estimation," IEEE Transactions on Biomedical Engineering, vol. 39, no. 7, pp. 665-675, July 1992.
[3] M. S. Hämäläinen and R. J. Ilmoniemi, "Interpreting magnetic fields of the brain: minimum norm estimates," Medical \& Biological Engineering \& Computing, vol. 32, no. 1, pp. 35-42, 1994.

[4] K. Brodmann, Vergleichende Lokalisationslehre der Grobhirnrinde, J.A.Barth, Leipzig, 1909.

[5] A. W. Toga, P. M. Thompson, S. Mori, et al., "Towards multimodal atlases of the human brain," Nature Reviews Neuroscience, vol. 7, no. 12, pp. $952-966,2006$.

[6] H. P. O. de Beeck, J. Haushofer, and N. G. Kanwisher, "Interpreting fMRI data : maps, modules and dimensions," Nature Reviews Neuroscience, vol. 9, no. 2, pp. 123-135, 2008.

[7] P. Rakic, "Neurocreationism-making new cortical maps," Science, vol. 294, no. 5544, pp. 1011-1012, 2001.

[8] O. Sporns, D. R. Chialvo, M. Kaiser, et al., "Organization, development and function of complex brain networks," Trends in Cognitive Sciences, vol. 8, no. 9, pp. 418-425, 2004.

[9] A.-C. Philippe, M. Clerc, T. Papadopoulo, et al., "A nested cortex parcellation combining analysis of MEG forward problem and diffusion MRI tractography," in IEEE International Symposium on Biomedical Imaging (ISBI), Barcelona, May 2012, pp. 518-521.

[10] B. Belaoucha, M. Clerc, and T. Papadopoulo, "Cortical surface parcellation via dMRI using mutual nearest neighbor condition," in IEEE International Symposium on Biomedical Imaging (ISBI), Prague, Czech Republic, Apr. 2016, pp. 903-906.

[11] D. G. Wakeman and R. N. Henson, "A multi-subject, multi-modal human neuroimaging dataset," Scientific Data, 2015.

[12] A. M. Dale, B. Fischl, and M. I. Sereno, "Cortical surface-based analysis I: Segmentation and surface reconstruction," NeuroImage, vol. 9, pp. 179-194, 1999.

[13] S. M. Smith, M. Jenkinson, M. W. Woolrich, et al., "Advances in functional and structural MR image analysis and implementation as FSL," NeuroImage, vol. 23, Supplement 1, pp. S208-S219, 2004, Mathematics in Brain Imaging.

[14] A. Gramfort, T. Papadopoulo, E. Olivi, et al., "OpenMEEG: opensource software for quasistatic bioelectromagnetics," BioMedical Engineering OnLine, vol. 9, no. 1, pp. 1-20, 2010.

[15] J. Kybic, M. Clerc, T. Abboud, et al., "A common formalism for the integral formulations of the forward EEG problem," Medical Imaging, IEEE Transactions on, vol. 24, no. 1, pp. 12-28, Jan 2005.

[16] D. Bajusz, A. Rácz, and K. Héberger, "Why is tanimoto index an appropriate choice for fingerprint-based similarity calculations?," Journal of Cheminformatics, vol. 7, no. 1, pp. 1-13, 2015.

[17] S. Arlot and A. Celisse, "A survey of cross-validation procedures for model selection," Statist. Surv., vol. 4, pp. 40-79, 2010.

[18] N. Kanwisher and J. Barton, "The functional architecture of the face system: Integrating evidence from fMRI and patient studies," in Oxford Handbook of Face Perception. Oxford University Press, 2011.

[19] J. V. Haxby, M. I. Gobbini, M. L. Furey, et al., "Distributed and overlapping representations of faces and objects in ventral temporal cortex," Science, vol. 293, no. 5539, pp. 2425-2430, 2001. 